\title{
Impact of Dielectric Constant on the Singlet-Triplet Gap in Thermally Activated Delayed Fluorescence Materials
}

\author{
Haitao Sun ${ }^{\dagger, \ddagger} *$, Zhubin $\mathrm{Hu}^{\dagger}$, Cheng Zhong ${ }^{\ddagger}$, Xiankai Chen ${ }^{\ddagger, \pi}$, \\ Zhenrong Sun $^{\dagger, \S}$, and Jean-Luc Brédas ${ }^{\ddagger \uparrow, \pi}$ \\ ${ }^{\dagger}$ State Key Laboratory of Precision Spectroscopy \\ School of Physics and Materials Science \\ East China Normal University \\ Shanghai 200062, P. R. China \\ ‡ Laboratory for Computational and Theoretical Chemistry of Advanced Materials \\ Physical Science and Engineering Division \\ King Abdullah University of Science and Technology \\ Thuwal 23955-6900, Kingdom of Saudi Arabia \\ ${ }^{\S}$ Collaborative Innovation Center of Extreme Optics \\ Shanxi University \\ Taiyuan, Shanxi 030006, P. R. China
}

* Corresponding authors: htsun@phy.ecnu.edu.cn; jean-luc.bredas@chemistry.gatech.edu

" New permanent address: School of Chemistry and Biochemistry, Georgia Institute of

Technology, Atlanta, Georgia 30332-0400 


\begin{abstract}
Thermally activated delayed fluorescence (TADF) relies on the presence of a very small energy gap, $\Delta E_{\mathrm{ST}}$, between the lowest singlet and triplet excited states. $\Delta E_{\mathrm{ST}}$ is thus a key factor in the molecular design of more efficient materials. However, its accurate theoretical estimation remains challenging, especially in the solid state due to the influence of polarization effects. In this Letter, we have quantitatively studied $\Delta E_{\mathrm{ST}}$ as a function of dielectric constant, $\varepsilon$, for four representative organic molecules using the methodology we recently proposed at the TammDancoff Approximation $\omega \mathrm{B} 97 \mathrm{X}$ level of theory, where the range-separation parameter $\omega$ is optimized with the polarizable continuum model (PCM). The results are found to be in very good agreement with experimental data. Importantly, the polarization effects can lead to a marked reduction in the $\Delta E_{\mathrm{ST}}$ value, which is favorable for TADF applications. This $\Delta E_{\mathrm{ST}}$ decrease in the solid state is related to the hybrid characters of the lowest singlet and triplet excited states, whose dominant contribution switches to charge-transfer-like with increasing $\varepsilon$. The present work provides a theoretical understanding on the influence of polarization effect on the singlet-triplet gap and confirms our methodology to be a reliable tool for the prediction and development of novel TADF materials.
\end{abstract}

\title{
TOC Graphic
}

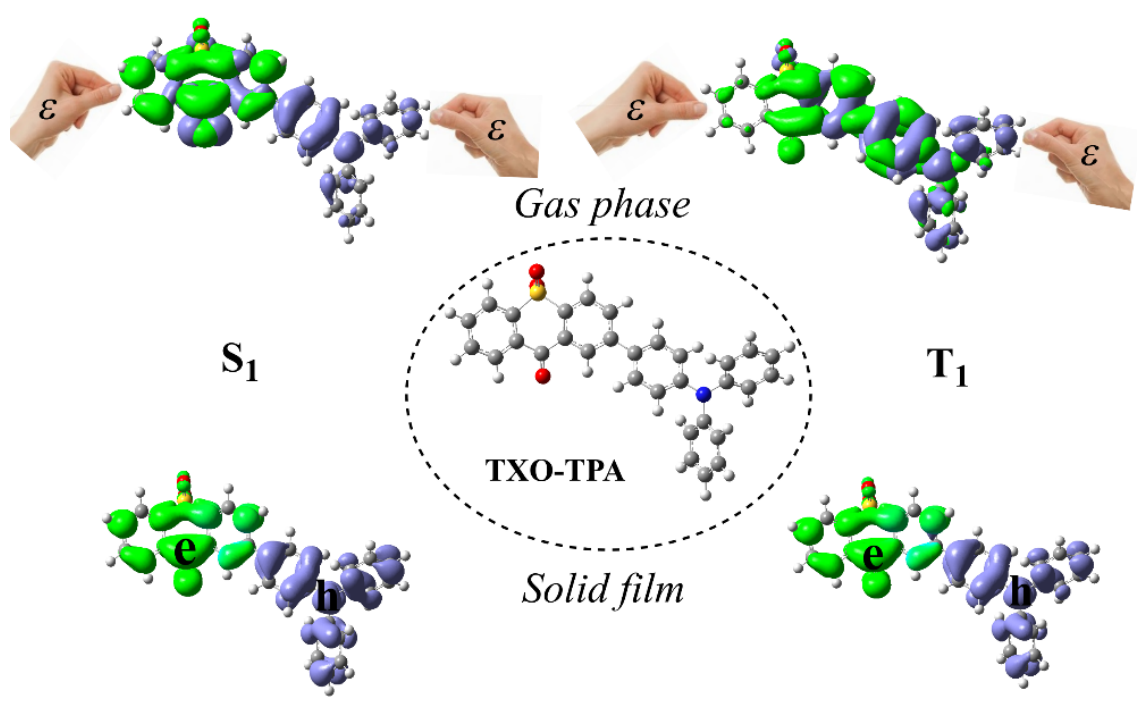


Organic light-emitting diodes (OLEDs) have attracted a great deal of attention due to their applications in high-resolution, ultra-thin displays for smart phones and televisions and in solidstate lighting. ${ }^{1,2}$ Recently, considerable efforts to develop new-generation, high-efficiency purely organic luminous materials have resulted in the exploitation of an emission mechanism based on thermally activated delayed fluorescence (TADF). ${ }^{3-7}$ Ideally, TADF allows the harvest of both singlet and triplet excitons and the realization of internal quantum efficiencies up to $100 \%$ in fluorescent OLEDs, which is comparable to those in phosphorescent OLEDs. ${ }^{8}$ Highly efficient TADF materials require a very small energy gap $\left(\Delta E_{\mathrm{ST}}\right)$, usually less than a hundred $\mathrm{meV}$, between the lowest singlet $\left(\mathrm{S}_{1}\right)$ and triplet $\left(\mathrm{T}_{1}\right)$ excited states, which allows for thermally activated up-conversion of the triplet excitons into $S_{1}$ via enhanced $T_{1} \rightarrow S_{1}$ reverse intersystem crossing (RISC). ${ }^{3,9}$ Thus, an accurate evaluation of $\Delta E_{\mathrm{ST}}$ is required in order to guide molecular design and expand the range of efficient TADF materials.

Simplistic considerations have often been used in the context of TADF molecules, as it has been expressed that very small $\Delta E_{\mathrm{ST}}$ values can be obtained when there is vanishing overlap between the highest occupied molecular orbital (HOMO) and lowest unoccupied molecular orbital (LUMO), under the assumption that both $\mathrm{S}_{1}$ and $\mathrm{T}_{1}$ correspond to HOMO-LUMO transitions. However, if such were the case, the oscillator strength between $S_{1}$ and $S_{0}$ (which primarily depends on wavefunction overlap) would go to zero and no luminescence would be detected; therefore, careful molecular design should be performed to strike the right balance and combine a small $\Delta E_{\mathrm{ST}}$ with a reasonable oscillator strength. In addition, a fact $\mathrm{T}_{1} \rightarrow \mathrm{S}_{1}$ reverse intersystem crossing rate is desired, which is related to the magnitude of spin-orbit coupling. ${ }^{10}$ 
It is well-established that the optical properties of $\pi$-conjugated organic molecules can vary considerably as a function of their environment. ${ }^{11}$ In the context of TADF, Méhes et al. found that there occur large variations in the photoluminescent (PL) quantum yield and PL transient decay time of a TADF guest molecule as a function of the nature of the host matrix, with the singlet-triplet gap $\Delta E_{\mathrm{ST}}$ shown to be closely related to the dipole moment or polarization of the matrix. ${ }^{12}$ Monkman et al. have shown that the TADF emitter and host combination must be optimized to minimize the reverse intersystem crossing barrier and maximize TADF efficiency. ${ }^{13}$ Very recently, Rajamalli et al. have reported that the same TADF compound can have much higher PL quantum yield in the solid state than that in solution. ${ }^{14}$ Thus, it is important to be able to describe accurately the excited-state properties of TADF emitters in the condensed phase, in particular, the singlet-triplet gap. Also, Ma and co-workers have reported the significance of a hybrid local/charge-transfer character of $\mathrm{S}_{1}{ }^{15}$, but these authors did not discuss the solid-state polarization effects that can play a key role in determining the nature of the states.

Theoretical studies of TADF molecules taking into account a solid-state environment have so far been limited. Our group recently developed a methodology, ${ }^{16-18}$ the polarizable continuum model $(\mathrm{PCM})^{19}$-tuned approach, in which optimal tuning of the range-separation parameter in a longrange corrected functional ${ }^{16,20-23}$ is combined with the description of the solid-state screening effects via the consideration of the solid-state dielectric constant $(\varepsilon)$ in the context of the PCM approach (see Computational Details in the Supporting Information, SI). Our original work has shown that the accuracy of this methodology in calculating the solid-state ionization potential (IP), electron affinity (EA), transport gap, polarization energy, and exciton binding energy values, is comparable to that of state-of-the-art many-body perturbation theory within the GW 
approximation, with the results in excellent agreement with experimental data. ${ }^{16-18}$ In this Letter, we apply this methodology to evaluate the singlet-triplet gap of four representative organic compounds (shown in Figure 1) as a function of the solid-state environment (for the sake of comparison, calculations were also performed with the widely used B3LYP functional).
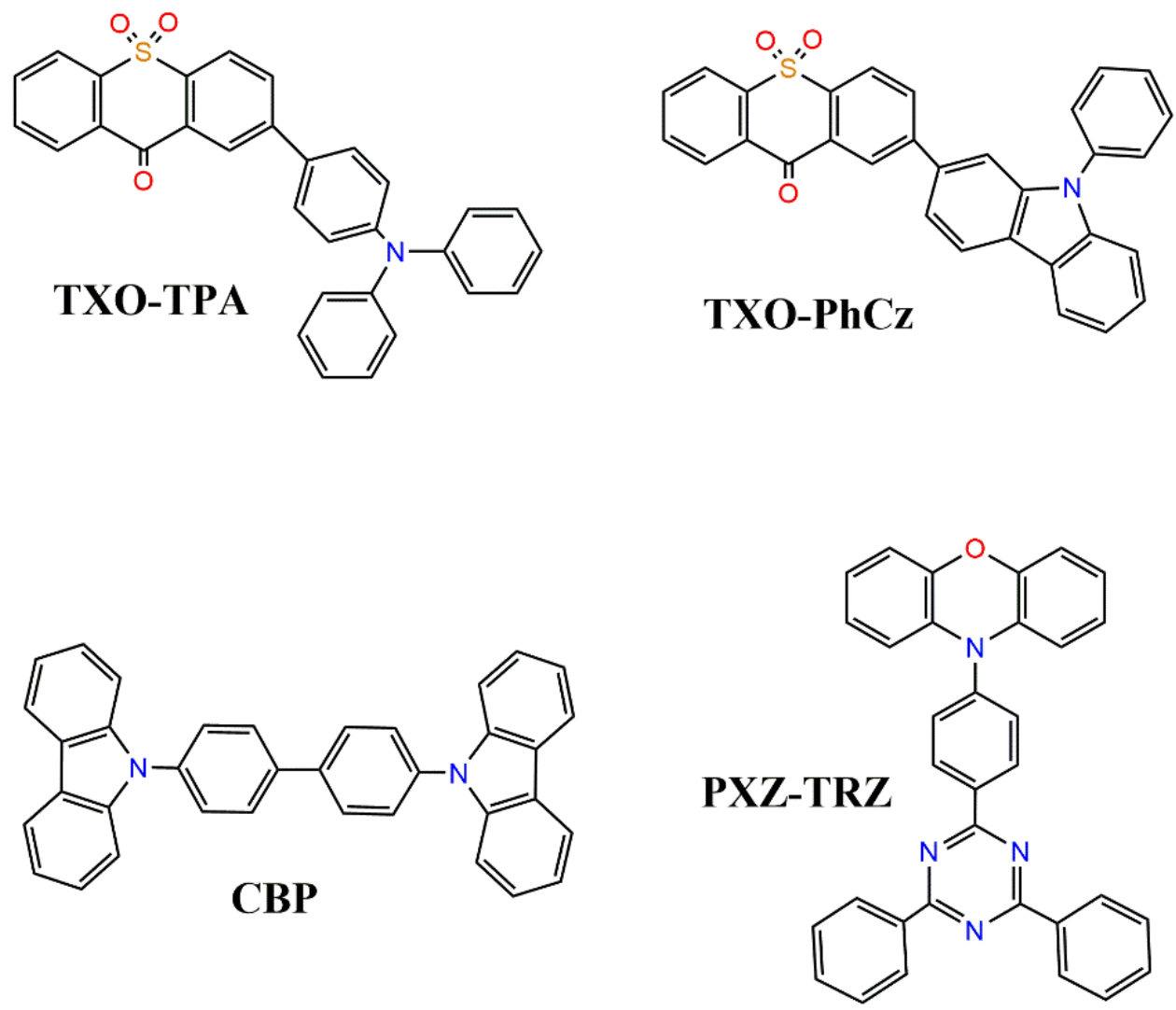

Figure 1. Chemical structures of the molecular materials studied in this work.

We have considered two TADF donor-acceptor-like molecules, TXO-TPA and TXO-PhCz, see Figure 1, which combine a 9-H-thioxanthen-9-one-10,10-dioxide (TXO) acceptor unit and either triphenylamine (TPA) or N-phenylcarbazole $(\mathrm{N}-\mathrm{PhCz})$ as a donor unit; these were taken from the 
work of Wang et al. ${ }^{7}$ In addition, we have investigated PXZ-TRZ (10-(4-(4,6-diphenyl-1,3,5triazin-2-yl)phenyl)-10H-phenoxazine), which has been reported to have a very small $\Delta E_{\mathrm{ST}}$ of $\sim 0.06 \mathrm{eV}^{6}$, as well as CBP (4,4'-bis(carbazol-9-yl)-p-biphenyl), which has a large $\Delta E_{\mathrm{ST}}$ of $\sim 0.71$ $\mathrm{eV}^{6}$ (we note that CBP is not a TADF molecule and is usually employed as a hole-transporting material in conventional OLEDs).

The ground-state geometries of the molecules were initially optimized at the B3LYP/cc-pVDZ level of theory. Then, the tuning procedure based on the range-separated (RS) functional $\left(\omega \mathrm{B} 97 \mathrm{X}^{24}\right)$ with the cc-pVDZ basis set was performed to obtain the optimal $\omega$ values for these geometries; the geometries were then reoptimized using the new $\omega$ values. This procedure was iterated until the change in $\omega$ became less than $10^{-3} \mathrm{Bohr}^{-1}{ }^{25}$ The vibrational frequencies of the optimized geometries were analyzed to ensure all positive frequencies were obtained. We found the optimal $\omega$ values were not sensitive to the ground-state geometries separately optimized by B3LYP and $\omega$ B97X functional. The optimal $\omega$ values are listed in Table 1. Hereafter, we refer to the optimally-tuned RS functional as $\omega \mathrm{B} 97 \mathrm{X}^{*}$, which was employed throughout this work. The vertical excitation (absorption) energies of the lowest singlet- $\left(E_{0}\left(\mathrm{~S}_{1}\right)\right)$ and triplet- $\left(E_{\mathrm{o}}\left(\mathrm{T}_{1}\right)\right)$ excited states and the vertical singlet-triplet gap $\left(\Delta E_{\mathrm{ST}}=E_{\mathrm{o}}\left(\mathrm{S}_{1}\right)-E_{\mathrm{o}}\left(\mathrm{T}_{1}\right)\right)$, and the adiabatic $\Delta E_{\mathrm{ST}^{*}}$ (the energy differences between the minima of the potential surfaces for $S_{1}$ and $T_{1}$ states), as depicted in Figure S1, have been calculated using the linear-response time-dependent density functional theory (TDDFT) ${ }^{26}$ with the Tamm-Dancoff approximation (TDA) $)^{27-29}$ at the $\omega \mathrm{B} 97 \mathrm{X} * / \mathrm{cc}-\mathrm{pVDZ}$ level. All calculations were performed using the Gaussian 09 software. $^{30}$ 
Table 1. Optimal range-separated parameters $\omega\left(\mathrm{Bohr}^{-1}\right)$, calculated ionization potential/electron affinity (IP/EA), transport gap $E_{g}$, lowest singlet and triplet excitation energy $\left(E_{0}\left(S_{1}\right)\right.$ and $\left.E_{0}\left(T_{1}\right)\right)$, oscillator strength $(f)$, exciton binding energy $\left(E_{b}\right)$, vertical and adiabatic singlet-triplet gap ( $\Delta \mathrm{E}_{\mathrm{ST}}$ and $\Delta \mathrm{E}_{\mathrm{ST}^{*}}$ ) (in $\mathrm{eV}$ ) of the molecules in both vacuum and solid phases at the (PCM-)tuned $\omega \mathrm{B} 97 \mathrm{X}^{*} / \mathrm{cc}-\mathrm{pVDZ}$ level. Experimental values are also listed in parentheses if available.

\begin{tabular}{|c|c|c|c|c|c|c|c|c|c|}
\hline Gas phase & & \multicolumn{8}{|c|}{$\omega \mathrm{B}^{2} \mathrm{X}^{*} / \mathrm{cc}-\mathrm{pVDZ}$} \\
\hline & $\omega$ & IP/EA & $E_{g}$ & $\mathrm{E}_{\mathrm{o}}\left(\mathrm{S}_{1}\right)$ & $f$ & $\mathrm{E}_{0}\left(\mathrm{~T}_{1}\right)$ & $\mathrm{E}_{\mathrm{b}}$ & $\Delta \mathrm{E}_{\mathrm{ST}}$ & $\Delta \mathrm{E}_{\mathrm{ST}^{*}}$ \\
\hline TXO-TPA & 0.159 & $6.67 / 1.10$ & 5.57 & 3.48 & 0.08 & 3.00 & 2.09 & 0.48 & 0.66 \\
\hline TXO-PhCz & 0.162 & $7.09 / 1.05$ & 6.04 & 3.50 & 0.00 & 3.03 & 2.54 & 0.47 & 0.58 \\
\hline CBP & 0.188 & $7.07 /-0.28$ & 7.35 & 4.29 & 1.18 & 3.43 & 3.06 & 0.86 & 1.10 \\
\hline PXZ-TRZ & 0.160 & $6.20 / 0.58$ & 5.62 & 3.10 & 0.05 & 3.02 & 2.52 & 0.08 & 0.12 \\
\hline Solid film & & \multicolumn{8}{|c|}{$\operatorname{PCM}(\varepsilon=3.0)$-tuned $\omega$ B97X*/cc-pVDZ } \\
\hline TXO-TPA & 0.041 & $\begin{array}{c}5.43 / 2.09 \\
(5.37 / 3.49)^{\mathrm{a}}\end{array}$ & 3.34 & $\begin{array}{c}2.17 \\
(2.06)^{\mathrm{b}} \\
\end{array}$ & 0.05 & 2.09 & 1.17 & 0.08 & $\begin{array}{c}0.06 \\
(0.05)^{c}\end{array}$ \\
\hline TXO-PhCz & 0.042 & $\begin{array}{c}5.83 / 2.07 \\
(5.78 / 3.58)^{\mathrm{a}}\end{array}$ & 3.76 & $\begin{array}{c}2.53 \\
(2.25)^{b}\end{array}$ & 0.03 & 2.43 & 1.23 & 0.10 & $\begin{array}{r}0.13 \\
(0.07)^{c} \\
\end{array}$ \\
\hline $\mathrm{CBP}$ & 0.027 & $5.41 / 0.83$ & 4.58 & 3.53 & 0.80 & 3.13 & 1.05 & 0.40 & 0.39 \\
\hline PXZ-TRZ & 0.042 & $5.01 / 1.47$ & 3.54 & 2.18 & 0.03 & 2.15 & 1.36 & 0.03 & 0.01 \\
\hline
\end{tabular}

${ }^{\mathrm{a}}$ The HOMO/LUMO levels determined from the onset of oxidation/reduction curves in cyclic voltammetry. ${ }^{b}$ Estimated from the onset of the absorption edge of the thin films. ${ }^{\mathrm{C}}$ Measured in TXO-TPA:mCP film and TXO-PhCz:mCP film, respectively.

As shown in Table 1 , the optimal $\omega$ values significantly reduce to roughly $0.16-0.19$ Bohr $^{-1}$ for the molecules in vacuum compared to the default $\omega=0.30 \mathrm{Bohr}^{-1}$ of the $\omega \mathrm{B} 97 \mathrm{X}$ functional. As TADF emitters, both TXO-TPA and TXO-PhCz were experimentally doped at a concentration of $5 \pm 1$ wt\% into a 1,3-bis(9H-carbazol-9-yl)benzene (mCP) host; ${ }^{7}$ thus, the solid-state environment can be considered to correspond to the dielectric constant of an amorphous mCP thin film, calculated to be $\sim 3.0$ (see Computational details, SI). For a dielectric constant of 3.0, the $\omega$ values decrease further to ca. 0.03-0.04 $\mathrm{Bohr}^{-1}$. Such a small $\omega$ value in the case of the simulated solid-state environment points to an electronic structure that is much more delocalized than in the 
gas phase, as a result of the inverse relationship between the tuned $\omega$ value and the spatial delocalization of the electron density. ${ }^{15,21}$

For the TXO-TPA molecule in the gas phase, $\Delta E_{\mathrm{ST}}\left[\Delta E_{\mathrm{ST}} *\right]$ is calculated to be $0.48[0.66] \mathrm{eV}$. When taking $\varepsilon=3, \Delta E_{\mathrm{ST}}\left[\Delta E_{\mathrm{ST}}{ }^{*}\right]$ of TXO-TPA is calculated to reduce to $0.08[0.06] \mathrm{eV}$. The calculated singlet-triplet gaps are found to be in excellent agreement with the experimental value of $0.05 \mathrm{eV}$ measured in a film. ${ }^{7}$ Similarly, for TXO-PhCz, the calculated $\Delta E_{\mathrm{ST}}\left[\Delta E_{\mathrm{ST}} *\right]$ values in the gas phase are $0.47[0.58] \mathrm{eV}$ and come down to $0.10[0.13] \mathrm{eV}$ when $\varepsilon=3$, also in good agreement with the experimental value of $0.07 \mathrm{eV}$. We note that Wang et al. utilized a moleculein-cluster scheme to estimate $\Delta E_{\mathrm{ST}}$, where the central molecule is treated at the quantummechanical (QM) level and the surrounding molecules in the cluster at the molecular mechanics (MM) level. ${ }^{7}$ The $\Delta E_{\mathrm{ST}}$ values calculated with this QM/MM approach are 0.08 and $0.16 \mathrm{eV}$ for TXO-TPA and TXO-PhCz, respectively, which is consistent with our results, (0.08 and $0.10 \mathrm{eV})$. The ionization potential (IP) calculated with our approach for TXO-TPA and TXO-PhCz are 5.43 and $5.83 \mathrm{eV}$, respectively, in excellent agreement with the experimental estimates of 5.37 eV (TXO-TPA) and 5.78 eV (TXO-PhCz) measured using cyclic voltammetry. The discrepancy between the calculated and measured values of electron affinity (EA) is relatively large. However, it is worth keeping in mind that much caution must be exercised when extrapolating solution electrochemical data to the solid state. ${ }^{31}$ The calculated lowest singlet excitation energies $E_{0}\left(S_{1}\right)$ for TXO-TPA and TXO-PhCz are 2.17 and $2.53 \mathrm{eV}$, respectively, which correspond well to the measured values of 2.06 and $2.25 \mathrm{eV}$ from the onset of the absorption edge in the thin films. The exciton binding energies, $E_{b}$, defined as the difference between the transport gap $\left(E_{g} \equiv I P-E A\right)$ and the optical gap in vacuum are in the range of 2.1 2.5 eV for TXO-TPA and TXO-PhCz; 
these $\mathrm{E}_{\mathrm{b}}$ values decrease to $\sim 1.2 \mathrm{eV}$ when $\varepsilon=3$, due to the solid-state polarization effects that screen the hole-electron interactions.

(a)

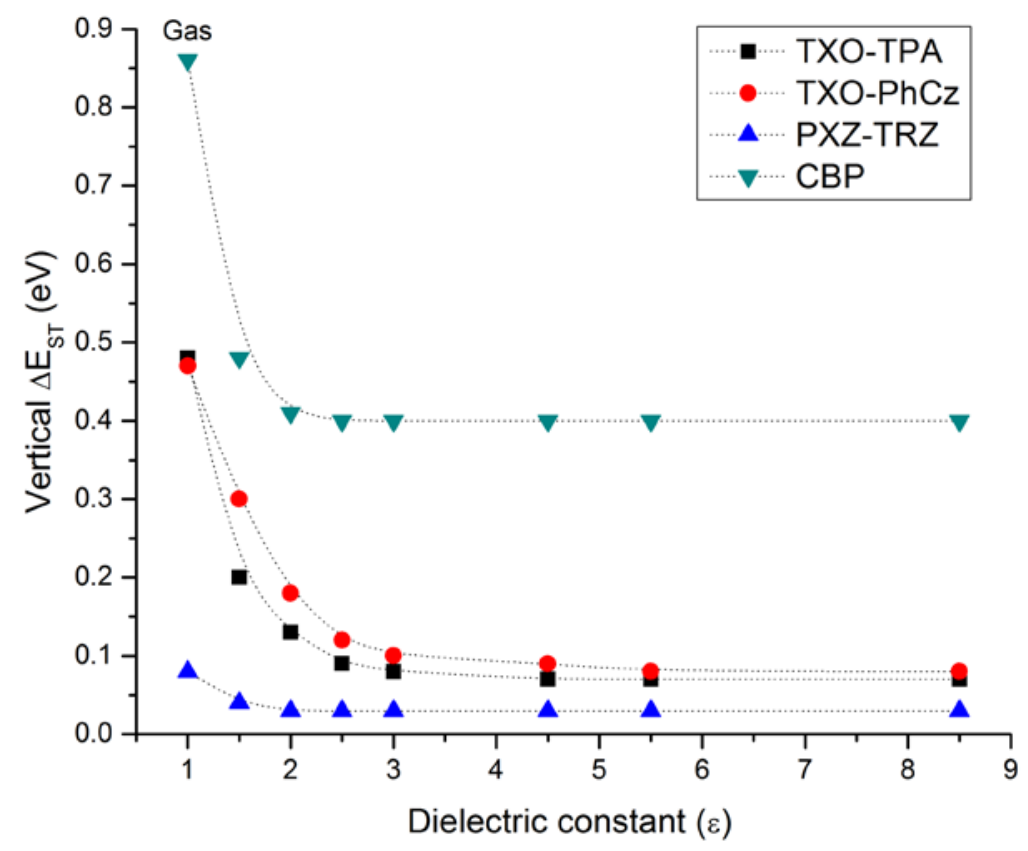

(b)

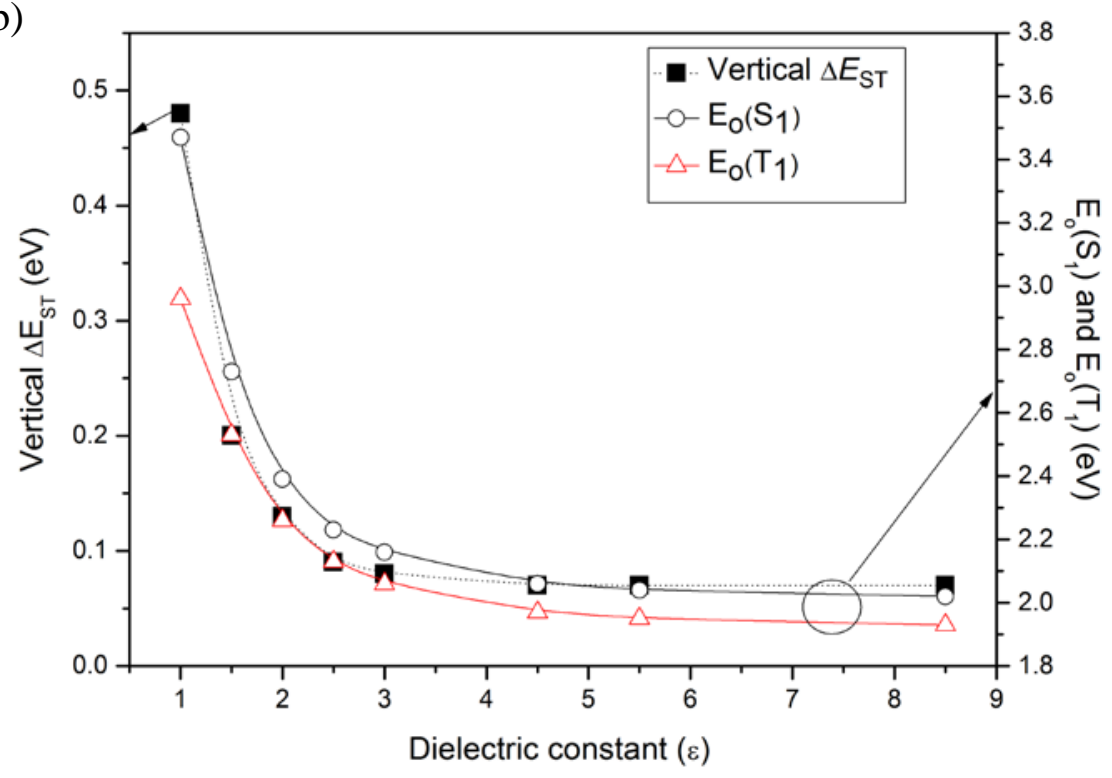

Figure 2. (a) Calculated vertical $\Delta E_{\mathrm{ST}}$ of TXO-TPA, TXO-PhCz, CBP, and PXZ-TRZ as a function of dielectric constant. (b) Calculated $\mathrm{E}_{0}\left(\mathrm{~S}_{1}\right), \mathrm{E}_{0}\left(\mathrm{~T}_{1}\right)$ and vertical $\Delta E_{\mathrm{ST}}$ of TXO-TPA as a function of dielectric constant. 
Table 2. Hole-electron wavefunction overlap $\left(O_{h, e}\right)$ and distance between the centroids of the hole and electron distributions $(\Delta r)$ in the lowest singlet and triplet states $\left(\mathrm{S}_{1}\right.$ and $\mathrm{T}_{1}$ ) for the four molecules in both vacuum and solid $(\varepsilon=3.0)$ phases. Calculations were performed at the (PCM)tuned $\omega \mathrm{B} 97 \mathrm{X}^{*} / \mathrm{cc}-\mathrm{pVDZ}$ level using high quality grids in the Multiwfn software ${ }^{32}$.

\begin{tabular}{|c|c|c|c|c|c|c|}
\hline & \multicolumn{3}{|c|}{ TXO-TPA } & \multicolumn{3}{|c|}{ TXO-PhCz } \\
\hline & $O_{h, e}$ & $\Delta r(\AA)$ & Character & $O_{h, e}$ & $\Delta r(\AA)$ & Character \\
\hline Gas phase $S_{1}$ & 0.24 & 2.40 & $\pi \pi^{*}\left({ }^{1} \mathbf{C T}+{ }^{1} \mathrm{LE}\right)+\mathbf{n} \pi^{*}$ & 0.23 & 1.27 & $\mathrm{n} \pi^{*}$ \\
\hline Gas phase $T_{1}$ & 0.36 & 2.86 & $\pi \pi^{*}\left({ }^{3} \mathbf{L E}+{ }^{3} \mathrm{CT}\right)$ & 0.23 & 1.24 & $\mathbf{n} \pi^{*}$ \\
\hline Solid film $\mathrm{S}_{1}$ & 0.05 & 8.66 & $\pi \pi^{*}\left({ }^{1} \mathrm{CT}+{ }^{1} \mathrm{LE}\right)$ & 0.06 & 7.66 & $\pi \pi^{*}\left({ }^{1} \mathbf{C T}+{ }^{1} \mathrm{LE}\right)$ \\
\hline \multirow[t]{3}{*}{ Solid film $\mathrm{T}_{1}$} & 0.05 & 8.66 & $\pi \pi^{*}\left({ }^{3} \mathrm{CT}+{ }^{3} \mathrm{LE}\right)$ & 0.09 & 7.06 & $\pi \pi^{*}\left({ }^{3} \mathbf{C T}+{ }^{3} \mathrm{LE}\right)$ \\
\hline & \multicolumn{3}{|r|}{ CBP } & \multicolumn{3}{|c|}{ PXZ-TRZ } \\
\hline & $O_{h, e}$ & $\Delta r(\AA)$ & Character & $O_{h, e}$ & $\Delta r(\AA)$ & Character \\
\hline Gas phase $S_{1}$ & 0.38 & 0.00 & $\pi \pi^{*}\left({ }^{1} \mathbf{L E}\right)$ & 0.05 & 5.20 & $\pi \pi^{*}\left({ }^{1} \mathbf{C T}\right)$ \\
\hline Gas phase $T_{1}$ & 0.56 & 0.00 & $\pi \pi^{*}\left({ }^{3} \mathbf{L E}\right)$ & 0.04 & 5.16 & $\pi \pi^{*}\left({ }^{3} \mathbf{C T}\right)$ \\
\hline Solid film $\mathrm{S}_{1}$ & 0.24 & 0.00 & $\pi \pi^{*}\left({ }^{1} \mathbf{L E}+{ }^{1} \mathrm{CT}\right)$ & 0.03 & 6.96 & $\pi \pi^{*}\left({ }^{1} \mathbf{C T}\right)$ \\
\hline Solid film $\mathrm{T}_{1}$ & 0.41 & 0.00 & $\pi \pi^{*}\left({ }^{3} \mathbf{L E}+{ }^{3} \mathrm{CT}\right)$ & 0.04 & 6.40 & $\pi \pi^{*}\left({ }^{3} \mathrm{CT}\right)$ \\
\hline
\end{tabular}

As shown in Figure 2a and Figure S2, the $\Delta E_{\mathrm{ST}}$ and $\Delta E_{\mathrm{ST}}{ }^{*}$ values of TXO-TPA and TXO-PhCz decrease significantly as the dielectric constant increases from 1.0 (vacuum) to 8.5. While the singlet-triplet gap has a non-TADF-character $\left(\Delta E_{\mathrm{ST}} \sim 0.5 \mathrm{eV}\right)$ in vacuum, it acquires TADF relevance $\left(\Delta E_{\mathrm{ST}} \sim 0.1 \mathrm{eV}\right)$ when $\varepsilon$ reaches $\sim 3$. As shown in Figure 2b, this $\Delta E_{\mathrm{ST}}$ decrease is related to the fact that the lowest singlet excited state, $\mathrm{S}_{1}$, has a ${ }^{1} \mathrm{CT}$ character, which is more sensitive to the polarization environment than the lowest triplet excited state, $\mathrm{T}_{1}$, which possesses a more local excitation $\left({ }^{3} \mathrm{LE}\right)$ character. To support our analysis, we plotted the hole and electron wavefunctions for the $\mathrm{S}_{1}$ and $\mathrm{T}_{1}$ states of TXO-TPA and TXO-PhCz in both vacuum and solid state, see Figure 3. To gain a more quantitative understanding, parameters such as the holeelectron wavefunction overlap $\left(O_{h, e}\right)$ and the distance $(\Delta r)^{33}$ between the centroids of the hole and electron distribution for the lowest singlet and triplet states were also calculated; they are 
listed in Table 2, together with the dominant character of each excited state. A smaller $O_{h, e}$ value and a larger $\Delta r$ value generally point to a stronger charge-transfer character.

For the $S_{1}$ state of TXO-TPA in the gas phase, the electron localizes on the TXO group with a wavefunction showing a typical $\pi^{*}$ character. The hole delocalizes along the backbone of molecule and displays both $\pi$ character and $\mathrm{n}(\mathrm{O})$ character. This results in an $O_{h, e}$ value of 0.24 and $\Delta r$ of $2.40 \AA . \mathrm{S}_{1}$ is made of significant $\pi \pi^{*}\left({ }^{1} \mathrm{CT}\right)$ and $n \pi^{*}$ transitions. The e-h overlap on the TXO moiety contributes to the oscillator strength of 0.08 (Table 1). For the $T_{1}$ state in vacuum, the hole and electron mainly localize along the central part of the molecule, which corresponds to a greater ${ }^{3} \mathrm{LE}$ character with an $O_{h, e}$ value of 0.36 and $\Delta r$ of $2.86 \AA$. However, in the solid state ( $\varepsilon$ = 3.0), the calculated $O_{h, e}$ and $\Delta r$ values for both $S_{1}$ and $T_{1}$ states become 0.05 and $8.66 \AA$, respectively, which demonstrates a significantly stronger CT character than in the gas phase. The small $\mathrm{e}-\mathrm{h}$ overlap around the middle of the molecule is responsible for the oscillator strength of 0.05. Interestingly, the $\mathrm{T}_{1}$ state of TXO-TPA switches from dominant ${ }^{3} \mathrm{LE}$ in the gas phase to a more ${ }^{3} \mathrm{CT}$ character in the solid state. This evolution is related to the increased $\mathrm{e}-\mathrm{h}$ screening (and decreased exciton binding energy) due to the medium polarization effect.

For TXO-PhCz in the gas phase, there is significant n-to- $\pi^{*}$ transition character for both $\mathrm{S}_{1}$ and $\mathrm{T}_{1}$ states that have vanishing $f$ values. However, when $\varepsilon=3.0$, the calculated $O_{h, e}[\Delta r]$ values are $0.06[7.66 \AA]$ for the $S_{1}$ state and $0.09[7.06 \AA]$ for the $T_{1}$ state; both values confirm a significant CT character, similar to the case of TXO-TPA. The small singlet-triplet gaps obtained when considering the polarizable medium point to TXO-TPA and TXO-PhCz as potentially efficient 
TADF materials, which would not be the case if one were to consider exclusively the results of the calculations in vacuum. Equally important is the fact that the $\mathrm{e}-\mathrm{h}$ overlap on the more centrally located benzene of the TXO unit of the two molecules provides oscillator strength to the $\mathrm{S}_{1}-\mathrm{S}_{0}$ transition.

For the sake of comparison, the singlet-triplet gaps of CBP and PXZ-TRZ were computed at the same (PCM-)tuned $\omega \mathrm{B} 97 \mathrm{X} * / \mathrm{cc}-\mathrm{pVDZ}$ level. CBP, a representative hole-transport material, has large $\Delta E_{\mathrm{ST}}(0.86 \mathrm{eV})$ and $\Delta E_{\mathrm{ST}} *(1.10 \mathrm{eV})$ values in the gas phase, which reduce to ca. $0.40 \mathrm{eV}$ in the thin-film environment, with substantial oscillator strength of 0.80 . As seen in Figure S3, the dominant characters in both $S_{1}$ and $T_{1}$ states remain of LE type even in the solid state. The calculated $O_{h, e}$ values are relatively large, in the range of $0.24 \sim 0.56$, and the $\Delta r$ values are all zero due to symmetry. Such large singlet-triplet gaps make CBP inappropriate for TADF applications. In the case of PXZ-TRZ, we calculate $\Delta E_{\mathrm{ST}}\left[\Delta E_{\mathrm{ST}}{ }^{*}\right]$ values of 0.08 [0.12] eV in the gas phase and $0.03[0.01] \mathrm{eV}$ in the solid state, which confirms the relevance of this molecule as an excellent TADF emitter. The calculated $O_{h, e}$ values are as small as $0.03 \sim 0.05$ and the $\Delta r$ values as large as $5.16 \sim 6.96 \AA$, pointing to significant CT characters in both gas phase and solid state. PXZ-TRZ possesses a small singlet-triplet gap $(<0.1 \mathrm{eV})$ in vacuum and the consideration of the polarization effects has little influence on $\Delta E_{\mathrm{ST}}$ (Figure 2a). Since the $\mathrm{S}_{1}$ and $\mathrm{T}_{1}$ states of PXZ-TRZ molecule both possess significant CT character in vacuum (Figure S3), they are stabilized equally upon polarization. 


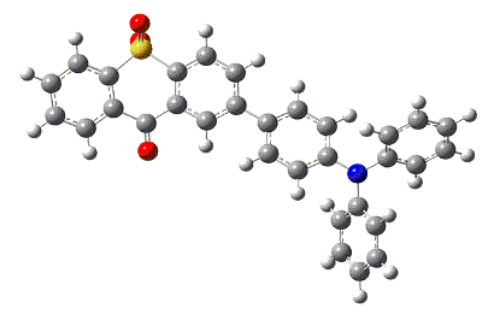

TXO-TPA

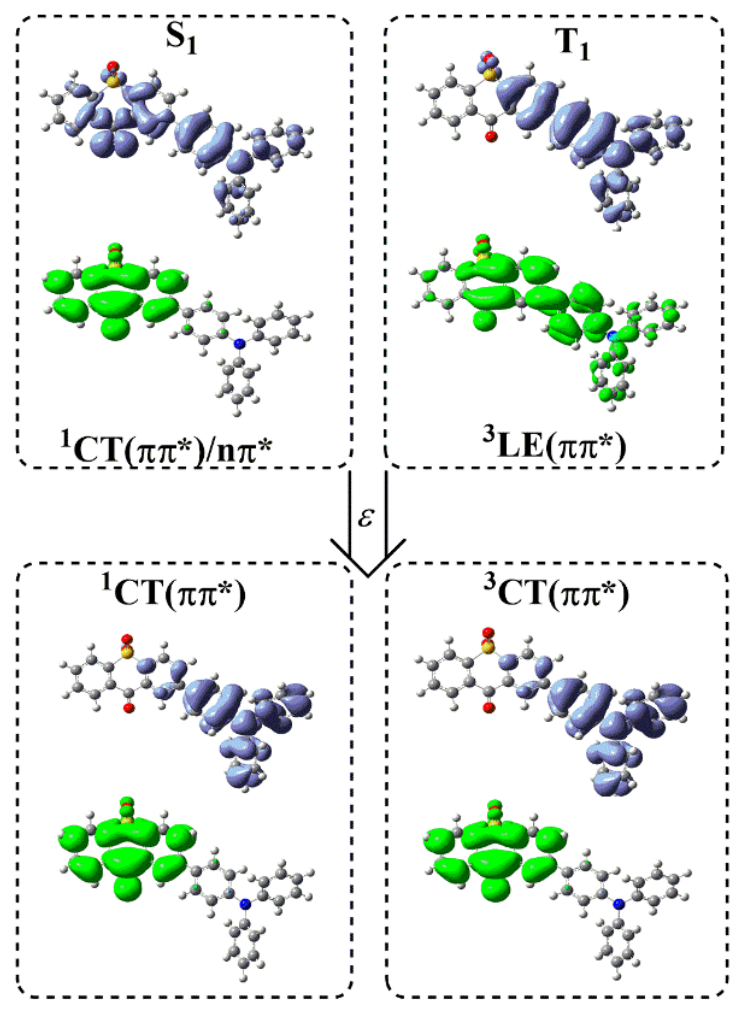

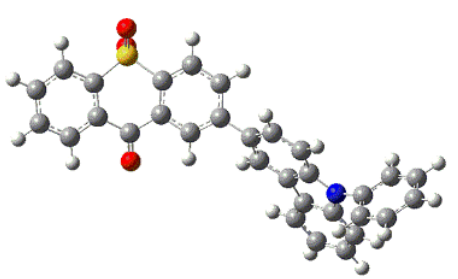

TXO-PhCz

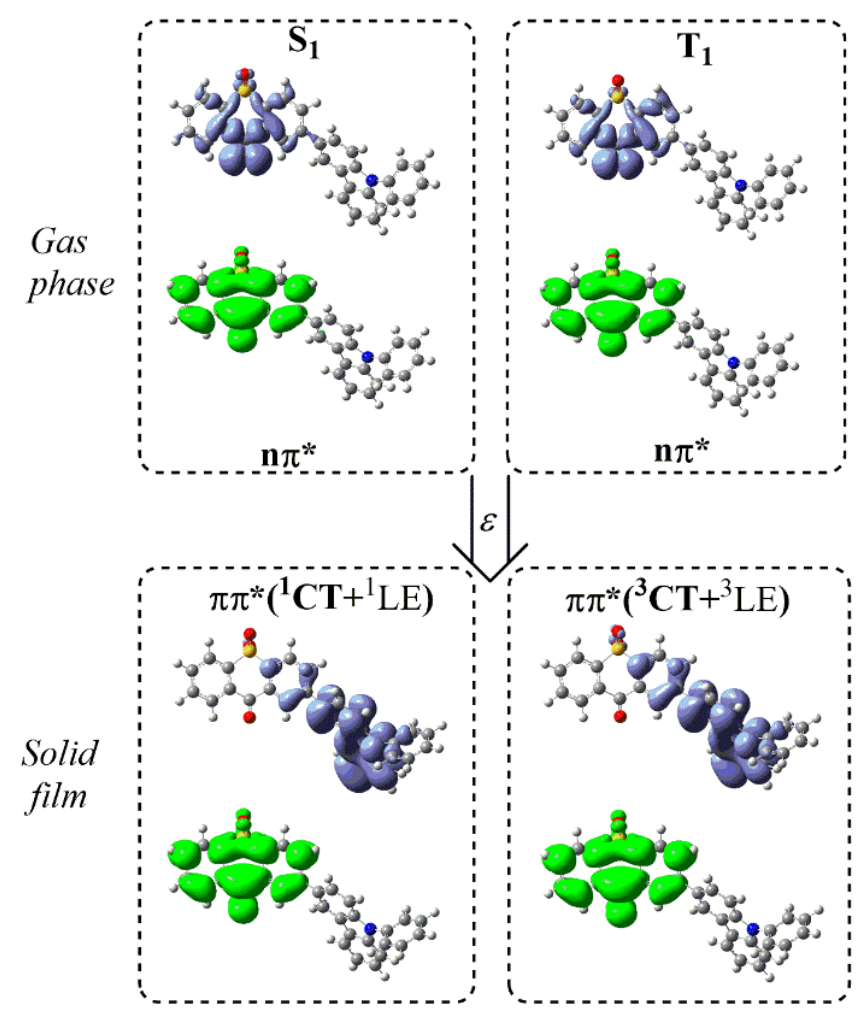

Figure 3. Distributions of hole and electron wavefunctions in the lowest singlet $\left(\mathrm{S}_{1}\right)$ and triplet state $\left(\mathrm{T}_{1}\right)$ of TXO-TPA and TXO-PhCz in both gas phase and solid film $(\varepsilon=3.0)$, as calculated at the PCM-tuned $\omega \mathrm{B} 97 \mathrm{X} * / \mathrm{cc}-\mathrm{pVDZ}$ level. Blue and green isosurfaces (iso. val. for density $=$ 0.0004) refer to hole and electron, respectively. CT and LE denote charge-transfer and localized excitations, respectively. Superscripts "1" or " 3 " indicate a singlet or triplet state. The dominant character of the excited state is indicated in bold. 
Finally, it is useful to compare the $\Delta E_{\mathrm{ST}}$ values of the TXO-TPA and PXZ-TRZ systems, calculated using the PCM-tuned method and the widely employed B3LYP functional. As shown in Figure 4, for both TXO-TPA and PXZ-TRZ, when using the B3LYP functional, the calculated $\Delta E_{\text {ST }}$ values remain practically unchanged as the dielectric constant increases. It is worth noting that the B3LYP-calculated $\Delta E_{\mathrm{ST}}$ values become reasonable only when describing the solid-state properties, in which case they agree well with the values obtained with the PCM-tuned $\omega \mathrm{B} 97 \mathrm{X}^{*}$ methodology when the dielectric constant is larger than $\varepsilon=2.0$. This can be attributed to the large electron self-interaction error of the B3LYP functional, which spuriously favors the CT character of the excitations and as a result implicitly includes the polarization effect. This finding is also consistent with a recent study by Phillips et al. ${ }^{34}$ However, the gas-to-solid change in singlet-triplet gaps cannot be captured by the B3LYP functional.

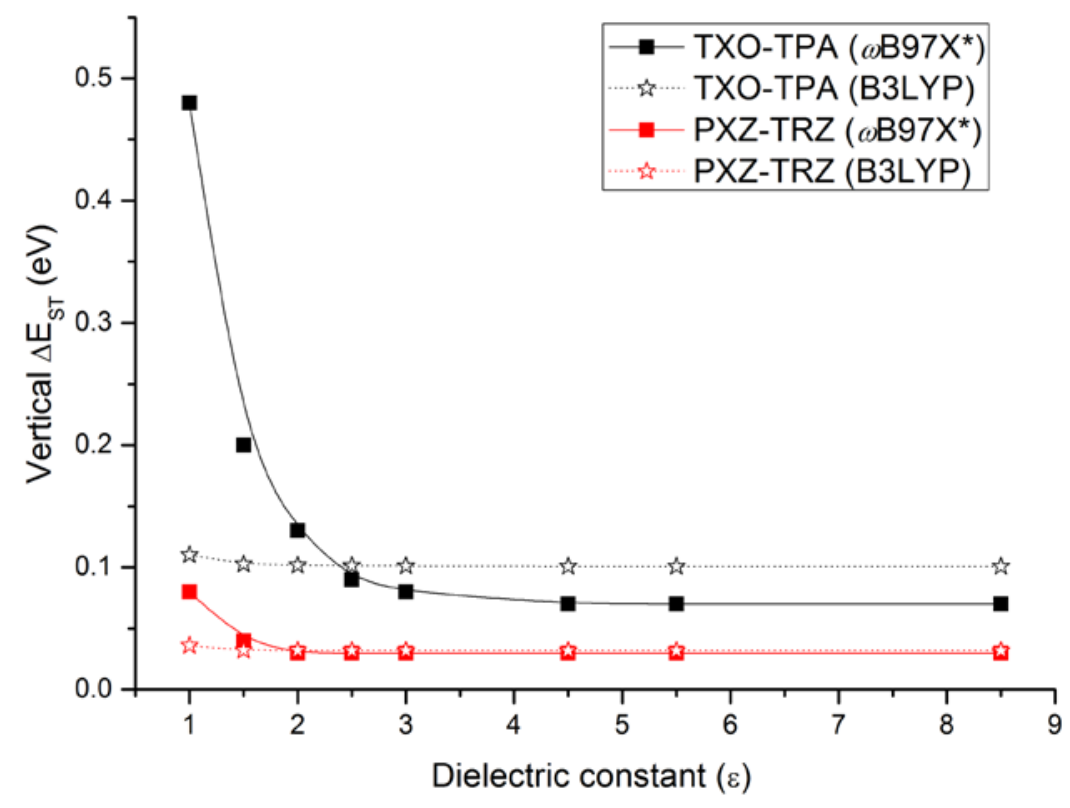

Figure 4. Calculated vertical $\Delta E_{\mathrm{ST}}$ for TXO-TPA and PXZ-TRZ as a function of dielectric constant using the (PCM-)tuned $\omega$ B97X* and B3LYP functionals. 
To summarize, we have calculated the vertical and adiabatic singlet-triplet gaps of four organic molecular materials (TXO-TPA, TXO-PhCz, CBP and PXZ-TRZ) at the TDA-PCM-tuned$\omega \mathrm{B} 97 \mathrm{X} * / \mathrm{cc}-\mathrm{pVDZ}$ level. The present work provides a theoretical understanding on the influence of polarization effects on the singlet-triplet gap of TADF molecules. The results indicate that:

(i) The singlet-triplet gap of TXO-TPA and $\mathrm{TXO}-\mathrm{PhCz}$ molecules in vacuum are reduced by an order of magnitude when embedded in a polarizable environment, indicating they are potentially efficient TADF materials. The calculated $\Delta E_{\mathrm{ST}}$ values using the PCM-tuned method are found to be in excellent agreement with the experimental values.

(ii) The small $\Delta E_{\mathrm{ST}}$ is attributed to the simultaneous presence of dominant ${ }^{1} \mathrm{CT}$ and ${ }^{3} \mathrm{CT}$ excited states due to the solid-state polarization. Since actual TADF applications require a non-negligible oscillator strength for the $\mathrm{S}_{1} \rightarrow \mathrm{S}_{0}$ transition in the solid state; this can be achieved by constructing a hybrid $S_{1}$ state combining both CT and LE characters through careful molecular design.

(iii) The widely used B3LYP functional gives reasonable results only when describing the singlet-triplet gaps in the solid state (because of its inherent strong electron selfinteraction error) and fails to describe the gas-to-solid phase evolution of the singlettriplet gap.

Notably, the singlet-triplet gap of molecules such as TXO-TPA and TXO-PhCz is very sensitive to the medium dielectric constant in the range $\varepsilon=1 \sim 4$; thus, great attention should be paid to the dielectric property of the host when doping TADF molecules in host-guest films. Current work in our groups is exploring the impact of various host materials and their detailed intermolecular interactions with TADF dopants in films. 


\section{Acknowledgments}

This work has been supported by the National Natural Science Foundation of China (Award No. 21603074 and 11474096), Shanghai-International Scientific Cooperation Fund (16520721200), Program of Introducing Talents of Discipline to Universities 111 Project (B12024), and King Abdullah University of Science and Technology (KAUST). We are very grateful to the ECNU and KAUST IT Research Computing Teams for providing assistance and computational and storage resources. H.S. thanks Prof. Bing Yang, Prof. Qisheng Zhang, Dr. Paul Winget, and Dr. Ko Inada for stimulating discussions.

\section{Supporting Information Available:}

Computational details regarding the PCM-tuned methodology described in this work and the calculation of the dielectric constant $\varepsilon$ of an amorphous mCP thin film; schematic representation of vertical $\Delta E_{\mathrm{ST}}$ and adiabatic $\Delta E_{\mathrm{ST}} *$ using potential energy surfaces; calculated adiabatic $\Delta E_{\mathrm{ST}} *$ of four molecular materials as a function of dielectric constant; distributions of hole and electron wavefunctions in the lowest singlet and triplet state of CBP and PXZ-TRZ in both gas phase and solid film. 


\section{References:}

(1) Tang, C. W.; VanSlyke, S. A., Organic Electroluminescent Diodes, Appl. Phys. Lett. 1987, 51, 913-915.

(2) Tremblay, J. The Rise of OLED Displays. Chem. Eng. News. 2016, 94, 30-34.

(3) Uoyama, H.; Goushi, K.; Shizu, K.; Nomura, H.; Adachi, C. Highly Efficient Organic Light-Emitting Diodes from Delayed Fluorescence. Nature, 2012, 492, 234-238.

(4) Hirata, S.; Sakai, Y.; Masui, K.; Tanaka, H.; Lee, S. Y.; Nomura, H.; Nakamura, N.; Yasumatsu, M.; Nakanotani, H.; Zhang, Q.; Shizu, K.; Miyazaki, H.; Adachi, C. Highly efficient blue electroluminescence based on thermally activated delayed fluorescence. Nat. Mater., 2015, 14, 330-336.

(5) Nakanotani, H.; Higuchi, T.; Furukawa, T.; Masui, K.; Morimoto, K.; Numata, M.; Tanaka, H.; Sagara, Y.; Yasuda, T.; Adachi, C. High-efficiency Organic Light-Emitting Diodes with Fluorescent Emitters. Nat. Commun., 2014, 5, 4016.

(6) Tanaka, H.; Shizu, K.; Nakanotani, H.; Adachi, C. Twisted Intramolecular Charge Transfer State for Long-Wavelength Thermally Activated Delayed Fluorescence. Chem. Mater., 2013, 25, 3766-3771.

(7) Wang, H.; Xie, L.; Peng, Q.; Meng, L.; Wang, Y.; Yi, Y.; Wang, P. Novel Thermally Activated Delayed Fluorescence Materials-Thioxanthone Derivatives and Their Applications for Highly Efficient OLEDs. Adv. Mater., 2014, 26, 5198-5204.

(8) Friend, R. H.; Gymer, R. W.; Holmes, A. B.; Burroughes, J. H.; Marks, R. N.; Taliani, C.; Bradley, D. D. C.; Santos, D. A. D.; Bredas, J. L.; Logdlund, M.; Salaneck, W. R. Electroluminescence in conjugated polymers. Nature, 1999, 397, 121-128.

(9) Sun, H.; Zhong, C.; Brédas, J.-L. Reliable Prediction with Tuned Range-Separated Functionals of the Singlet-Triplet Gap in Organic Emitters for Thermally Activated Delayed Fluorescence. J. Chem. Theory Comput., 2015, 11, 3851-3858.

(10) Samanta, P.; Kim, D.; Coropceanu, V.; Brédas, J.-L. Up-Conversion Intersystem Crossing Rates in Organic Emitters for Thermally Activated Delayed Fluorescence: Impact of the Nature of Singlet vs Triplet Excited States. J. Am. Chem. Soc., 2017, 139, 4042-4051.

(11) Varghese, S.; Das, S. Role of Molecular Packing in Determining Solid-State Optical Properties of $\pi$-Conjugated Materials. J. Phys. Chem. Lett., 2011, 2, 863-873.

(12) Méhes, G.; Goushi, K.; Potscavage Jr, W. J.; Adachi, C. Influence of Host Matrix on Thermally-Activated Delayed Fluorescence: Effects on Emission Lifetime, Photoluminescence Quantum Yield, and Device Performance. Org. Electron., 2014, 15, 2027-2037.

(13) dos Santos, P. L.; Ward, J. S.; Bryce, M. R.; Monkman, A. P. Using Guest-Host Interactions To Optimize the Efficiency of TADF OLEDs. J. Phys. Chem. Lett. 2016, 7, 33413346. 
(14) Rajamalli, P.; Senthilkumar, N.; Gandeepan, P.; Huang, P.-Y.; Huang, M.-J.; Ren-Wu, C.-Z.; Yang, C.-Y.; Chiu, M.-J.; Chu, L.-K.; Lin, H.-W.; Cheng, C.-H. A New Molecular Design Based on Thermally Activated Delayed Fluorescence for Highly Efficient Organic Light Emitting Diodes. J. Am. Chem. Soc., 2016, 138, 628-634.

(15) Zhang, S.; Yao, L.; Peng, Q.; Li, W.; Pan, Y.; Xiao, R.; Gao, Y.; Gu, C.; Wang, Z.; Lu, P.; Li, F.; Su, S.; Yang, B.; Ma,Y. Achieving a Significantly Increased Efficiency in Nondoped Pure Blue Fluorescent OLED: A Quasi - Equivalent Hybridized Excited State. Adv. Funct. Mater. 2015, 25, 1755-1762.

(16) Sun, H.; Ryno, S.; Zhong, C.; Ravva, M.; Sun, Z.; Körzdörfer, T.; Brédas, J.-L. Ionization Energy, Electron Affinity, and Polarization Energy of Organic Molecular Crystals: Quantitative Estimations from a Polarizable Continuum Model (PCM)-Tuned Range-Separated Density Functional Approach. J. Chem. Theory Comput., 2016, 12, 2906-2916.

(17) Sun, H.; Hu, Z.; Zhong, C.; Zhang, S.; Sun, Z. Quantitative Estimation of Exciton Binding Energy of Polythiophene-Derived Polymers using Polarizable Continuum Model-Tuned Range-Separated Density Functional. J. Phys. Chem. C. 2016, 120, 8048-8055.

(18) Hu, Z.; Zhou, B.; Sun, Z.; Sun, H. Prediction of Excited-state Properties of Oligoacene Crystals Using Polarizable Continuum Model-Tuned Range-separated Hybrid Functional Approach, J. Comput. Chem., 2017, 38, 569-575.

(19) Tomasi, J.; Mennucci, B.; Cammi, R. Quantum Mechanical Continuum Solvation Models. Chem. Rev., 2005, 105, 2999-3094.

(20) Baer, R.; Livshits, E.; Salzner, U. Tuned Range-Separated Hybrids in Density Functional Theory. Annu. Rev. Phys. Chem., 2010, 61, 85-109.

(21) Kronik, L.; Stein, T.; Refaely-Abramson, S.; Baer, R. Excitation Gaps of Finite-Sized Systems from Optimally Tuned Range-Separated Hybrid Functionals. J. Chem. Theory Comput., 2012, 8, 1515-1531.

(22) Sun, H.; Autschbach, J. Influence of the Delocalization Error and Applicability of Optimal Functional Tuning in Density Functional Calculations of Nonlinear Optical Properties of Organic Donor-Acceptor Chromophores. ChemPhysChem, 2013, 14, 2450-2461.

(23) Sun, H.; Autschbach, J. Electronic Energy Gaps for $\pi$-Conjugated Oligomers and Polymers Calculated with Density Functional Theory. J. Chem. Theory Comput., 2014, 10, 10351047.

(24) Chai, J.-D.; Head-Gordon, M. Systematic Optimization of Long-range Corrected Hybrid Density Functionals. J. Chem. Phys., 2008, 128, 084106.

(25) Tamblyn, I.; Refaely-Abramson, S.; Neaton, J. B.; Kronik, L. Simultaneous Determination of Structures, Vibrations, and Frontier Orbital Energies from a Self-Consistent Range-Separated Hybrid Functional. J. Phys. Chem. Lett. 2014, 5, 2734-2741. 
(26) Runge, E.; Gross, E. K. U. Density-Functional Theory for Time-Dependent Systems. Phys. Rev. Lett. 1984, 52, 997-1000.

(27) Hirata, S.; Head-Gordon, M. Time-dependent Density Functional Theory Within the Tamm-Dancoff Approximation. Chem. Phys. Lett., 1999, 314, 291-299.

(28) Peach, M. J. G.; Williamson, M. J.; Tozer, D. J. Influence of Triplet Instabilities in TDDFT. J. Chem. Theory Comput., 2011, 7, 3578-3585.

(29) Peach, M. J. G.; Tozer, D. J. Overcoming Low Orbital Overlap and Triplet Instability Problems in TDDFT. J. Phys. Chem. A, 2012, 116, 9783-9789.

(30) Frisch, M. J.; Trucks, G. W.; Schlegel, H. B.; Scuseria, G. E.; Robb, M. A.; Cheeseman, J. R.; Scalmani, G.; Barone, V.; Mennucci, B.; Petersson, G. A. and et al. Gaussian 09 Revision D.01. 2009.

(31) Brédas, J.-L. Mind the gap! Mater. Horiz., 2014, 1, 17-19.

(32) Lu, T.; Chen, F. Multiwfn: A Multifunctional Wavefunction Analyzer. J. Compt. Chem., 2012, 33, 580-592.

(33) Guido, C. A.; Cortona, P.; Mennucci, B.; Adamo, C. On the Metric of Charge Transfer Molecular Excitations: A Simple Chemical Descriptor. J. Chem. Theory Comput., 2013, 9, 31183126.

(34) Phillips, H.; Zheng, Z.; Geva, E.; Dunietz, B. D. Orbital Gap Predictions for Rational Design of Organic Photovoltaic Materials. Org. Electron. 2014, 15, 1509-1520. 